\title{
Caracterización clínico-patológica de la esofagitis eosinofílica en niños y adolescentes en el Hospital Universitario Fundación Santa Fe de Bogotá
}

\section{Clinical Pathology Characterization of Eosinophilic Esophagitis in Children and Adolescents at Hospital Universitario Fundación Santa Fe de Bogotá}

\author{
Leonardo F. Jurado, ${ }^{1}$ Jennifer Arroyave-Benavides, ${ }^{2}$ Ana Jiménez-Uribe, ${ }^{2}$ José Fernando Vera-Chamorro, ${ }^{*}{ }^{*}$ Carolina Zambrano-Pérez, ${ }^{2}$
} Rocío del Pilar López-Panqueva. ${ }^{4}$

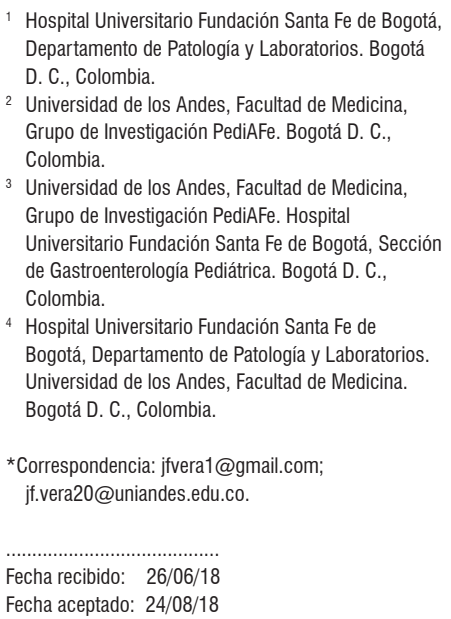

3 Universidad de los Andes, Facultad de Medicina, Grupo de Investigación PediAFe. Hospital Universitario Fundación Santa Fe de Bogotá, Sección de Gastroenterología Pediátrica. Bogotá D. C. Colombia.

${ }^{4}$ Hospital Universitario Fundación Santa Fe de Bogotá, Departamento de Patología y Laboratorios. Universidad de los Andes, Facultad de Medicina. Bogotá D. C., Colombia.

*Correspondencia: jfvera1@gmail.com; jf.vera20@uniandes.edu.co.

Fecha recibido: $\quad 26 / 06 / 18$ Fecha aceptado: 24/08/18

\begin{abstract}
Resumen
Introducción: la esofagitis eosinofilica (EEo) es una enfermedad emergente, crónica e inmunomediada. Clínicamente se caracteriza por síntomas asociados con disfunción esofágica e histológicamente por infiltrado inflamatorio predominante de eosinófilos. Objetivo: describir las características clínicas, endoscópicas e histopatológicas de niños y adolescentes con diagnóstico de EEo en el Hospital Universitario Fundación Santa Fe de Bogotá entre 2007 y 2017. Métodos: estudio observacional descriptivo de corte transversal que incluyó pacientes menores de 18 años con diagnóstico histopatológico de EEo. Resultados: se incluyeron 46 pacientes, 31 de sexo masculino, con una edad promedio de 11,8 años (rango: 11 meses-18 años). El $70 \%$ presentó dolor abdominal, el $37 \%$ pirosis, $28 \%$ emesis y el $22 \%$ náuseas y disfagia. Los antecedentes más referidos fueron asma $(41 \%)$, rinitis alérgica $(37 \%)$, enfermedad por reflujo gastroesofágico $(22 \%)$ y dermatitis atópica $(15 \%)$. El hallazgo endoscópico más frecuente fue los exudados blanquecinos, descritos en el $35 \%$. La sospecha endoscópica de EEo se describió en el $50 \%$ de los casos. El estudio histopatológico mostró de 15 a 40 eosinófilos por campo de alto poder (CAP) en el $52 \%, 41-60$ en el 19,5 \% y $61-80$ en el 15,2\%. Se encontró hiperplasia de la lámina basal en el $95,6 \%$ de los casos. Conclusiones: los pacientes en su mayoría eran adolescentes (69 $\%$ ), el síntoma más frecuente fue el dolor abdominal $(70 \%$ y el $40 \%$ de los casos tenía antecedente de atopia. Solamente el 50 \% tenía hallazgos endoscópicos sugestivos de EEo. El presente trabajo corresponde al primer análisis clínico y patológico de casos de EEo en niños y adolescentes en Colombia.
\end{abstract}

\section{Palabras clave}

Esofagitis eosinofilica, eosinófilos, patología, niños, adolescentes, Colombia (fuente: BIREME).

\begin{abstract}
Introduction: Eosinophilic esophagitis $(\mathrm{E} o \mathrm{E})$ is an emerging, chronic and immune-mediated disease. Clinically it is characterized by symptoms associated with esophageal dysfunction, and histologically by predominantly inflammatory eosinophil infiltrate. Objective: The aim of this study was to describe the clinical, endoscopic and histopathological characteristics of children and adolescents diagnosed with EoE at the Hospital Universitario Fundación Santa Fe de Bogotá between 2007 and 2017. Methods: This is a cross-sectional, descriptive and observational study that included patients under 18 years of age with histopathological diagnoses of EoE. Results: Forty-six patients were included, 31 were male, and the average age was 11.8 years (range 11 months - 18 years). Seventy percent presented abdominal pain, $37 \%$ presented heartburn, $28 \%$ suffered vomiting, $22 \%$ had nausea and dysphagia. The most common antecedents were asthma (41\%), allergic rhinitis (37\%), gastroesophageal reflux disease (22\%) and atopic dermatitis (15\%). The most frequent endoscopic finding consisted of whitish exudates found in 35\% of the cases. Endoscopic suspicion of EoE was described in $50 \%$ of the cases. The histopathological study showed 15 to 40 eosinophils per high power field (HPF) in 52\%, 41-60/HPF in 19.5\%, and $61-80 / \mathrm{HPF}$ in $15.2 \%$. Hyperplasia of the basal lamina was found in $95.6 \%$ of the cases. Conclusions: The majority of patients were adolescents (69\%), the most frequent symptom was abdominal pain $(70 \%)$, and $40 \%$ of cases had histories of atopy. Only $50 \%$ had endoscopic findings suggestive of EoE. This study is the first clinical and pathological analysis of EoE cases in children and adolescents in Colombia.
\end{abstract}

\section{Keywords}

Eosinophilic esophagitis, eosinophils, pathology, children, adolescents, Colombia. (Source: Bireme). 


\section{INTRODUCCIÓN}

La esofagitis eosinofílica (EEo) es una enfermedad emergente, inmunomediada y de crónica evolución que se caracteriza por infiltración de eosinófilos al epitelio esofágico, y en los casos no tratados resulta en fibrosis y disfunción esofágica $(1,2)$. Inicialmente considerada una entidad rara, actualmente es una de las condiciones más frecuentemente diagnosticadas en niños con problemas para la alimentación y en adultos con disfagia e impactación alimentaria (3).

La primera descripción clínica de EEo se reportó en 1968 (4). Inicialmente, se consideraba como una manifestación de la enfermedad por reflujo gastroesofágico (ERGE); sin embargo, en la década de los noventa se identificaron casos de EEo en niños y adultos que presentaban otras manifestaciones y en quienes los síntomas y las alteraciones histológicas no mejoraban con la supresión de producción de ácido, ni con cirugía antirreflujo. Posteriormente, otros estudios (5-7) informaron la resolución de la EEo en respuesta al manejo con dieta, hallazgos que sugirieron que se trataba de una entidad única, no siempre relacionada con $\operatorname{ERGE}(2,3)$.

Desde el 2000 se ha observado un exponencial aumento en la prevalencia de la EEo especialmente en países occidentales; actualmente, su incidencia anual se compara con la de la enfermedad de Crohn (8-11). No obstante, no es claro si la EEo es realmente una enfermedad nueva o simplemente de reciente reconocimiento (12).

La EEo se ha reportado en niños y adultos en todo el mundo, con una clara predominancia por el género masculino. Noel y colaboradores (13) reportaron una incidencia en niños en Ohio de 1/10 000 entre el 2000 y 2003, con una prevalencia de 4/10 000 para el 2003. Por su parte, Cherian y colaboradores (11) en 2006 en un estudio realizado en Australia informaron un aumento en la prevalencia de 0,05/10 000 en el año 1995 a 0,89/10 000 para el 2004.

Más recientemente, un estudio poblacional en Estados Unidos evaluó la prevalencia de esta entidad en más de 35 millones de registros clínicos generados entre 2008 y 2011. Se encontró que 16405 pacientes tenían al menos un código diagnóstico para EEo, el $24 \%$ era menor de 18 años y la prevalencia en menores de 20 años se calculó en $50,5 / 100000$ (14). En otro estudio que incluyó alrededor de 30 millones de registros clínicos generados entre 2010 y 2015 , se encontraron 7840 casos de EEo, de los cuales 1250 correspondían a pacientes pediátricos $(15,9 \%)$, lo que indica una prevalencia de 25,1/100 000 (15).

Se ha hecho evidente que después de la publicación de las recomendaciones del primer consenso internacional sobre EEo, el reconocimiento y oportunidad de diagnóstico de esta patología entre la comunidad médica ha mejorado sustancialmente (16). Este consenso definió por primera vez como criterio diagnóstico de EEo el hallazgo de más de 15 eosinófilos por CAP en el tejido esofágico $(3,16)$.

Tal como en el caso de otras enfermedades inmunomediadas, entre ellas el asma o el eccema, la EEo es considerada una enfermedad de evolución crónica $(17,18)$. Datos de estudios clínicos aleatorizados (19-21), así como cohortes prospectivas y retrospectivas $(22,23)$, han mostrado que la EEo no presenta tendencia a resolverse espontáneamente. Por una parte, en un estudio que realizó seguimiento a pacientes pediátricos durante un período de 14 años, solo el $2 \%$ de los participantes tuvo remisión de la enfermedad (24). Por otra parte, no existe evidencia de transformación de la EEo en síndrome hipereosinofílico, comprometiendo otras áreas del tracto gastrointestinal (TGI) o causando neoplasia (25).

La sospecha de EEo inicia con la presencia de síntomas asociados con disfunción o fibrosis esofágica $(2,3)$. La presentación y evolución de la enfermedad va a cambiar según la edad del paciente (17). Usualmente, los niños presentan síntomas relacionados con disfunción esofágica, que pueden simular ERGE. Los infantes menores de 5 años pueden presentar falla en el desarrollo, dificultades en la alimentación y atoramiento con comidas sólidas. Los niños de 6 a 14 años presentan vómito, dolor abdominal y disfagia, mientras que los síntomas relacionados con el desarrollo de estenosis esofágica y fibrosis, como impactación alimentaria y disfagia, son más frecuentes en los mayores de 15 años $(17,26)$. Adicionalmente, es común que los pacientes con diagnóstico de EEo también padezcan de otras condiciones atópicas como rinitis, dermatitis, asma, alergias alimentarias mediadas por inmunoglobulina $\mathrm{E}(\mathrm{IgE})$ e historia familiar de atopia, entre otras $(1,3)$.

Para el diagnóstico, además de una completa historia clínica, herramientas como la endoscopia y el estudio histológico del tejido esofágico son fundamentales (3). La sensibilidad y especificidad de la endoscopia en el diagnóstico de EEo es del $15 \%-48 \%$ y $90 \%-98 \%$, respectivamente (27). Los principales hallazgos endoscópicos reportados son la pérdida del patrón vascular normal de la mucosa, presencia de anillos corrugados, felinización de la mucosa (anillos concéntricos), exudados blancos, surcos longitudinales, estenosis esofágica, mucosa en papel crepé y anillos de constricción en los casos avanzados $(2,28)$.

El estudio histopatológico es actualmente el estándar de oro para el diagnóstico, aun cuando los eosinófilos en el esófago no son exclusivos de esta condición y no solo se encuentran asociados con la EEo, su conteo es fundamental en el diagnóstico $(29,30)$. Como se comentó, se ha establecido como punto de corte el hallazgo de $\geq 15$ eosinófilos por CAP. Una vez se realiza el diagnóstico, el control de la inflamación esofágica es imperativo, no solo para aliviar los síntomas, sino también para prevenir complicaciones como la estenosis esofágica $(2,3)$. 
El presente trabajo buscó describir y analizar restrospectivamente las características de presentación clínica y los hallazgos histopatológicos en pacientes menores de 18 años con diagnóstico de EEo atendidos en el Hospital Universitario Fundación Santa Fe (HUFSFB) entre enero de 2007 y octubre de 2017.

\section{PACIENTES Y MÉTODOS}

Se adelantó un estudio observacional descriptivo de corte transversal. Se inició con la revisión de las bases de datos de patología y endoscopia digestiva en busca de pacientes menores de 18 años con diagnóstico histológico de EEo (conteo de $\geq 15$ eosinófilos por CAP) en el HUFSFB entre enero de 2007 y octubre de 2017. Una vez se completó la revisión, el listado de pacientes se registró en Excel 2013. Posteriormente, se revisaron los reportes de los estudios de patología (conteo tisular de eosinófilos, evidencia de microabscesos, elongación papilar, cambios vasculares, acantosis, hiperplasia de la membrana basal, cambios fibróticos y eosinofilia gástrica y duodenal) y endoscopia (alteración del patrón vascular de la mucosa, presencia de exudados blanquecinos, presencia de anillos corrugados, anillos concéntricos, exudados blancos, surcos longitudinales, estenosis esofágica, mucosa en papel crepé y anillos de constricción).

Desde el sistema de historias clínicas del HUFSFB se recolectó la información clínica pertinente (motivo de consulta, reporte de dolor abdominal, náuseas, emesis, halitosis, pirosis, disfagia, regurgitación, tos, diarrea, sensación de saciedad temprana, impactación alimentaria, entre otros). Con el software STATA 11.0 se realizó un análisis estadístico descriptivo de la población. El protocolo del estudio contó con la revisión y aval del comité de ética en investigación del HUFSFB.

\section{RESULTADOS}

Durante el período de estudio se encontraron 46 pacientes con diagnóstico de EEo, la edad promedio fue 11,8 años (rango: 11 meses-18 años), 31 (67,39\%) eran de sexo masculino y la relación hombre: mujer fue de $2: 1$. Las características clínicas y antecedentes de la población se describen en la Tabla 1.

Los hallazgos endoscópicos esofágicos más frecuentemente encontrados fueron los exudados blanquecinos, con 16 casos (34,78\%), y los surcos longitudinales encontrados en 7 casos $(15,21 \%)$ (Figura 1). Considerando los antecedentes de síntomas digestivos asociados con enfermedad atópica, así como los hallazgos endoscópicos, en 23 pacientes se sospechó EEo (50\%), en 14 pacientes (30,43\%) se consideró esofagitis erosiva péptica y en 2 pacientes (4,34 \%) se sospechó esofagitis por Cándida spp (Tabla 2).
Tabla 1. Características generales y clínicas de los pacientes

\begin{tabular}{|c|c|c|}
\hline Variable & $\begin{array}{c}\text { Número } \\
n=46\end{array}$ & $\begin{array}{l}\text { Porcentaje } \\
100\end{array}$ \\
\hline \multicolumn{3}{|l|}{ Sexo } \\
\hline Hombre & 31 & 67,93 \\
\hline Mujer & 15 & 32,07 \\
\hline Edad promedio & 11,8 años & Rango: 11 meses-18 años \\
\hline \multicolumn{3}{|l|}{ Características clínicas } \\
\hline Dolor abdominal & 32 & 69,56 \\
\hline Pirosis & 17 & 36,95 \\
\hline Vómito & 12 & 26,08 \\
\hline Náuseas & 10 & 21,73 \\
\hline Disfagia & 10 & 21,73 \\
\hline Hipo & 6 & 13,04 \\
\hline Halitosis & 6 & 13,04 \\
\hline Impactación de comida & 4 & 8,69 \\
\hline Carraspeo & 4 & 8,69 \\
\hline Diarrea & 3 & 6,52 \\
\hline Tos & 3 & 6,52 \\
\hline Regurgitación & 2 & 4,34 \\
\hline $\begin{array}{l}\text { Sensación de saciedad } \\
\text { temprana }\end{array}$ & 2 & 4,34 \\
\hline Pérdida de peso & 1 & \\
\hline Falla en el crecimiento & 1 & \\
\hline \multicolumn{3}{|l|}{ Antecedentes } \\
\hline Asma & 19 & 41,30 \\
\hline Rinitis alérgica & 17 & 36,95 \\
\hline ERGE & 10 & 21,73 \\
\hline Dermatitis atópica & 7 & 15,21 \\
\hline Alergia alimentaria & 6 & 13,04 \\
\hline Tiroiditis de Hashimoto & 3 & 6,52 \\
\hline $\begin{array}{l}\text { Otros desórdenes } \\
\text { inmunomediados }\end{array}$ & 3 & 6,52 \\
\hline
\end{tabular}

Tabla 2. Hallazgos endoscópicos

\begin{tabular}{lcc}
\hline \multicolumn{1}{c}{ Variable } & $\begin{array}{c}\text { Número } \\
\mathbf{n}=\mathbf{4 6}\end{array}$ & $\begin{array}{c}\text { Porcentaje } \\
\mathbf{1 0 0}\end{array}$ \\
\hline Exudados blanquecinos & 16 & 34,78 \\
Surcos longitudinales & 7 & 15,21 \\
Cuerpo extraño & 3 & 6,52 \\
Características compatibles con gastritis & 33 & 71,73 \\
Ureasa para Helicobacter pylori positiva & 5 & 10,86 \\
Opinión del especialista & & \\
$\quad$ Esofagitis eosinofílica & 23 & 50 \\
$\quad$ Esofagitis erosiva péptica & 14 & 30,43 \\
Esofagitis por Candida spp. & 2 & 4,34 \\
\hline
\end{tabular}


Con el estudio histológico del tejido esofágico, en 24 pacientes $(52,17 \%)$ se encontró un conteo de eosinófilos por CAP de 15 a 40, 41 a 60 en 9 pacientes (19,56\%), 61 a 80 en 7 pacientes $(15,21 \%), 81$ a 100 en 1 paciente $(2,17 \%)$ y más de 100 eosinófilos por CAP en 5 pacientes $(10,86 \%)$. Los hallazgos histológicos adicionales más frecuentes fueron hiperplasia de la lámina basal en 44 pacientes $(95,65 \%)$, acantosis en 14 pacientes $(30,43 \%)$ y degranulación eosinofílica en 12 pacientes (26,08 \%) (Figura 2, Tabla 3).

\section{DISCUSIÓN}

Durante las últimas décadas se han hecho grandes avances en el entendimiento de la epidemiología y patogenia de la EEo (23) y también ha sido evidente un notable aumento en su incidencia y prevalencia en todo el mundo, probablemente relacionado con el mejor reconocimiento de esta entidad $(2,16)$.
Tabla 3. Hallazgos histológicos

\begin{tabular}{lcc}
\hline \multicolumn{1}{c}{ Variable } & $\begin{array}{c}\text { Número } \\
\mathbf{n = 4 6}\end{array}$ & $\begin{array}{c}\text { Porcentaje } \\
\mathbf{1 0 0}\end{array}$ \\
\hline Conteo de eosinófilos por CAP: & 24 & \\
15 a 40 & 92,17 \\
41 a 60 & 7 & 19,56 \\
61 a 80 & 1 & 15,21 \\
81 a 100 & 5 & 2,17 \\
Más de 100 & 44 & 10,86 \\
Hiperplasia de la lámina basal & 14 & 35,65 \\
Acantosis & 12 & 26,43 \\
Degranulación eosinofilica & 8 & 17,39 \\
Elongación papilar & 7 & 15,21 \\
Microabscesos & 4 & 8,69 \\
Infiltrado eosinofílico gástrico o duodenal & 31 & 67,39 \\
Hallazgos compatibles con gastritis crónica & 10 & 32,25 \\
Bacilos compatibles con H. pylori & \\
\hline
\end{tabular}
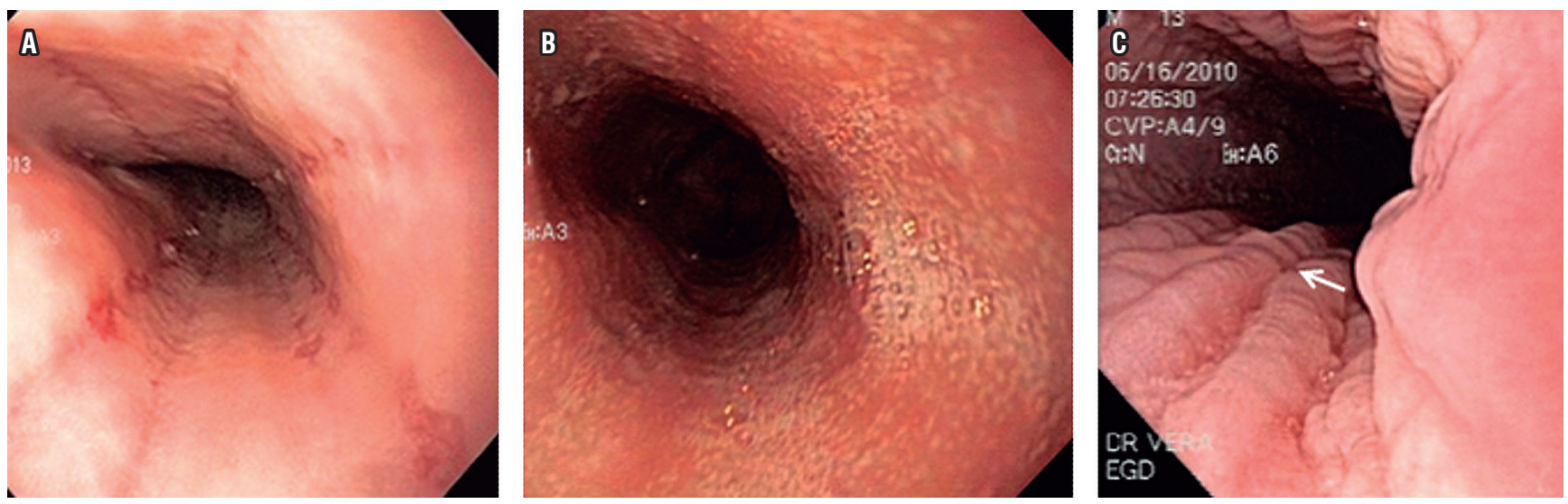

Figura 1. Esofagoscopia. A. surcos longitudinales. B. Exudados blanquecinos. C. Erosiones.
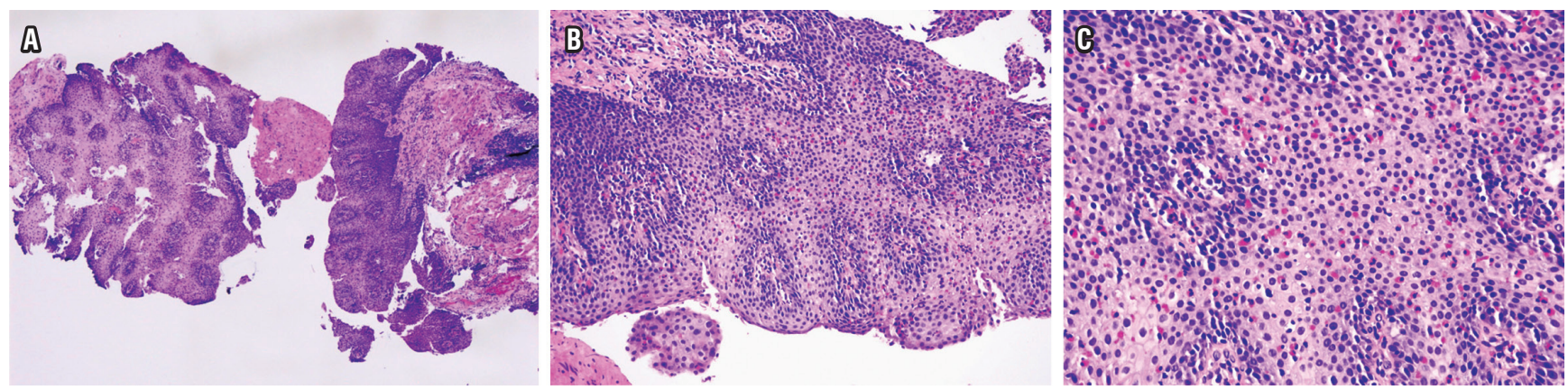

Figura 2. A. H \& E 4 X, muestra marcada acantosis, hiperplasia de células basales y papilomatosis. B. H \& E 20 X, muestra aumento de la celularidad intraepitelial, predominantemente de eosinófilos. C. H \& E 40 X, muestra infiltrado de eosinófilos con presencia de más de 80 por CAP. 
La EEo se ha reportado en cualquier etapa de la vida, desde el período de lactancia hasta los casi 100 años de edad (30, $31)$. No obstante, la mayoría de los casos se diagnostica en niños, adolescentes y adultos menores de 50 años $(10,26)$. Existe una constante discrepancia entre sexos, siendo 3 a 4 veces más frecuente en hombres que en mujeres, y también es más frecuente en individuos de raza blanca $(23,32)$.

Un estudio multicéntrico (33) incluyó a 214 pacientes de varios países latinoamericanos entre ellos Colombia y encontró que el $71 \%$ era de sexo masculino. En cuanto a la edad, el $2 \%$ correspondía a lactantes, $16 \%$ a preescolares, $35 \%$ a adolescentes y $47 \%$ a escolares. De manera similar, en la serie aquí descrita se encontró que la gran mayoría de pacientes era de sexo masculino; sin embargo, en contraste con los hallazgos de la cohorte latinoamericana (33), la edad al momento del diagnóstico más frecuente encontrada (69\%) fue la adolescencia ( 12 a 18 años).

De acuerdo con la evidencia acumulada, la historia natural de la EEo se caracteriza por 2 fases de presentación: una forma predominantemente inflamatoria (usualmente infantil), que puede evolucionar a una predominantemente fibrosante (de la adultez) (22). Esta información explica en parte las diferencias entre la presentación clínica de los niños frente a los adultos $(1,16,17)$.

Entonces se propone que, en los niños, la inflamación eosinofílica en el esófago se manifiesta con placas o exudados blanquecinos, alteración de la vasculatura o edema de la mucosa, y surcos lineales, pero ausencia de anillos de constricción, lo que causa síntomas como dolor, pirosis y, en algunos casos, falla del crecimiento. Cuando los eventos patogénicos progresan, usualmente en ausencia de tratamiento adecuado, se desarrolla fibrosis y anillos de constricción, que generan un patrón clínico caracterizado por disfagia en la adolescencia y adultez, aunque los anillos fibrosos también pueden ser vistos en niños $(1,3,26,28)$. Los hallazgos del presente estudio se correlacionan con lo reportado, siendo el síntoma más frecuentemente encontrado el dolor abdominal; seguido por pirosis, síntoma que suele verse más comúnmente en adolecentes (17), quienes constituyen la mayoría de nuestra población.

La evidencia muestra que los pacientes con diagnóstico de EEo presentan una tasa de condiciones atópicas superior a la de individuos sin EEo $(1,3,17)$. Se ha descrito que del $30 \%$ al $50 \%$ de los niños con EEo tienen asma, en comparación con el $10 \%$ de la población sin asma; de forma similar, del $50 \%$ al $75 \%$ padecen de rinitis alérgica, en comparación con el $30 \%$ de la población sin EEo. Entre el $10 \%$ y $20 \%$ de los infantes con EEo padecen de alergia alimentaria mediada por $\operatorname{IgE}$ (tipo urticaria y anafilaxis), en comparación con el $1 \%$ a $5 \%$ de los infantes normales $(2,17)$.

Son consistentes con estos datos las características de esta serie, en la que más del $40 \%$ de los pacientes tenía antece- dente de asma, el $37 \%$ de rinitis alérgica y $13 \%$ de alergia alimentaria. Adicionalmente, se encontraron antecedentes de otras condiciones inmunomediadas como tiroiditis de Hashimoto, vitiligo, angioedema y urticaria.

Con respecto a los hallazgos endoscópicos, las alteraciones más frecuentemente encontradas en esta serie fueron los exudados blanquecinos y los surcos longitudinales. Solamente en la mitad de los pacientes, considerando los hallazgos endoscópicos y las características clínicas, el especialista consideró a la EEo como diagnóstico endoscópico principal, lo cual demuestra la importancia de toma de biopsias.

La EEo es la única forma de enfermedad eosinofílica del TGI, a la cual se le ha definido un criterio objetivo para su diagnóstico. Se reconoce que los eosinófilos residen normalmente en la mucosa del TGI excepto en el esófago (34), por tanto, las enfermedades eosinofílicas son más fáciles de caracterizar en el esófago que en otra porción del TGI (35). Esto fue posible gracias a un estudio de seguimiento (25) en el cual se encuestó a un grupo de pacientes a quienes se les había encontrado eosinófilos en biopsias esofágicas practicadas 15 años atrás (durante la niñez). Inicialmente, se encontró que estos individuos, comparados con controles, reportaron más frecuentemente disfagia. Adicionalmente, los pacientes con un $\geq 15$ eosinófilos por CAP reportaron más frecuentemente el diagnóstico médico de alergia alimentaria, antecedente de impactación alimentaria y necesidad de seguimiento por gastroenterología, en comparación con los pacientes cuyo conteo tisular de eosinófilos era $<15$. También describen que aquellos pacientes con al menos 5 eosinófilos por CAP reportaron más frecuentemente impactación alimentaria en comparación con los controles, esto sugiere que un conteo inferior al adoptado (15 eosinófilos por CAP) tiene también significancia clínica. Los hallazgos de este estudio también demuestran que la intensidad de la infiltración eosinofílica epitelial se relaciona más frecuentemente con alteraciones tisulares en el epitelio y la lámina propia (25).

La presentación histológica de la EEo compromete todos los componentes tisulares representados en una biopsia. El epitelio puede verse acantósico debido a la expansión de la zona basal, la que puede corresponder a la totalidad del engrosamiento epitelial. La presencia de células inflamatorias intraepiteliales, en este caso eosinófilos, corresponde a la respuesta de células $\mathrm{T}$ ayudantes de tipo 2 (Th2) frente a los antígenos deglutidos (35). Además de la alteración numérica, los eosinófilos suelen presentarse con distribución anómala, en forma de exudado luminal y formación de microabscesos con exudado; o en el epitelio intacto, tapizando la superficie; o por el contrario, concentrarse más predominantemente en la lámina propia. Es posible observar degranulación de eosinófilos, posiblemente secundaria a disrupción celular mecánica (35). En contraste con la histología 
de una lámina propia normal, en los casos de EEo, sus fibras suelen estar engrosadas y densas (36), puede verse comprometida por inflamación eosinofílica crónica (18) y algunas veces con numerosas células plasmáticas (35).

De este modo, los hallazgos histológicos más frecuentemente descritos entre los pacientes de esta cohorte fueron la hiperplasia de la lámina basal, acantosis y degranulación de eosinófilos. Otros cambios como la elongación papilar y la formación de microabscesos fueron menos frecuentes. De manera interesante, en 31 pacientes (67\%) la biopsia gástrica mostró características histológicas compatibles con gastritis crónica, y en 10 de los pacientes (32\%) se observaron bacilos compatibles con $H$. pylori.

Respecto al conteo de eosinófilos por CAP, más de la mitad de los pacientes presentó un conteo entre 15 y 40 eosinófilos y 7 pacientes ( 2 niñas y 5 niños) tuvieron un conteo igual o superior a 80 eosinófilos. Resulta interesante observar que 6 de ellos eran preadolescentes mayores de 12 años; además, este grupo incluye a los 3 pacientes con antecedente de tiroiditis de Hashimoto y todos ellos presentaban diagnóstico de alguna condición atópica o alergia alimentaria. A pesar de que no es posible realizar un análisis más profundo, este hallazgo permite inferir respecto a la influencia de la edad del diagnóstico en la severidad de la presentación, así como la relevancia del perfil clínico atópico o inmunomediado entre los pacientes con diagnóstico de EEo.

Adicionalmente, durante la última década el interés en el estudio de la etiología y patogenia de la EEo ha permitido conocer más sobre esta condición $(2,23)$. Múltiples factores de riesgo se han descrito; sin embargo, varios estudios han demostrado que la infección por $H$. pylori podría ser un factor protector frente a la eosinofilia esofágica y otras enfermedades atópicas (37).

Un estudio analizó más de 165000 especímenes pareados de biopsias esofágicas y gástricas, y se encontró una fuerte asociación inversa entre H. pylori y la evidencia de eosinofilia esofágica; es decir, aquellos individuos con mayor riesgo de eosinofilia esofágica o diagnóstico de EEo tenían menor probabilidad de estar infectados con $\mathrm{H}$. pylori (38). Este hallazgo también se ha descrito en otros estudios de pacientes con diagnóstico de EEo (39) y es consistente con el hecho de que la infección por esta bacteria se relaciona inversamente con el desarrollo de otras enfermedades atópicas como el asma y el eccema (40). Aún se desconoce el mecanismo por el cual esta condición puede ser un factor protector para EEo, pero se sugiere que se trata de una polarización al sistema inmune hacia una respuesta de las células $\mathrm{T}$ ayudantes de tipo 1 (Th1), mientras que la ausencia de infección favorece la respuesta de las células Th2, disminuyendo la tolerancia y, por tanto, la atopia (38).

Al particularizar las características de los 10 pacientes en quienes se evidenció al $H$. pylori en la biopsia gástrica, no se encontró mayor diferencia en el conteo de eosinófilos (entre $20 \mathrm{y}>100$ por CAP) y al considerar el promedio (44,5 eosinófilos por CAP), este es muy similar al de la cohorte completa (41 eosinófilos por CAP). Con respecto a los antecedentes de atopia, en 3 de los 10 pacientes no se encontró ningún diagnóstico de enfermedad atópica.

El presente trabajo corresponde al primer análisis publicado de las características clínicas, endoscópicas e histopatológicas de la EEo en infantes y adolescentes adelantado en el país. Hallazgos como el predominio del sexo masculino son consistentes con lo descrito en otros países de Latinoamérica y el mundo; sin embargo, otros como la edad de los pacientes al momento del diagnóstico son disímiles frente a lo descrito en otras cohortes de pacientes.

Hubo también variación en la frecuencia de síntomas y hallazgos endoscópicos y patológicos. Fue claro que la presentación de la EEo es inespecífica, por lo que siempre es necesario un alto índice de sospecha, así como una buena correlación de los antecedentes clínicos, la sintomatología y el estudio endoscópico e histológico.

Hallazgos relacionados con las comorbilidades atópicas y otras condiciones inmunomediadas, así como la frecuencia de infección por $H$. pylori llaman la atención y demandan análisis más profundos que contribuyan a un mejor entendimiento de la forma de presentación de la EEo en Colombia.

\section{REFERENCIAS}

1. Liacouras CA, Furuta GT,Hirano I,Atkins D, Attwood SE, Bonis PA, et al. Eosinophilic esophagitis: updated consensus recommendations for children and adults. J Allergy Clin Immunol. 2011;128(1):3-20.e6. doi: 10.1016/j.jaci.2011.02.040.

2. Cianferoni A, Spergel J. Eosinophilic Esophagitis: A Comprehensive Review. Clin Rev Allergy Immunol. 2016;50(2):159-74. doi: 10.1007/s12016-015-8501-z.

3. Furuta GT, Katzka DA. Eosinophilic Esophagitis. N Engl J Med. 2015;373(17):1640-8. doi: 10.1056/ NEJMra1502863.

4. Hardy WR, Anderson RE. The hypereosinophilic syndromes. Ann Intern Med. 1968;68(6):1220-9.

5. Attwood SE, Smyrk TC, Demeester TR, Jones JB. Esophageal eosinophilia with dysphagia. A distinct clinicopathologic syndrome. Dig Dis Sci. 1993;38(1):109-16.

6. Straumann A, Spichtin HP, Bernoulli R, Loosli J, Vögtlin J. Idiopathic eosinophilic esophagitis: a frequently overlooked disease with typical clinical aspects and discrete endoscopic findings. Schweiz Med Wochenschr. 1994;124(33):1419-29.

7. Kelly KJ, Lazenby AJ, Rowe PC, Yardley JH, Perman JA, Sampson HA. Eosinophilic esophagitis attributed to gastroesophageal reflux: improvement with an amino acidbased formula. Gastroenterology. 1995;109(5):1503-12.

8. Prasad GA, Alexander JA, Schleck CD, Zinsmeister AR, Smyrk TC, Elias RM, et al. Epidemiology of eosinophilic esophagitis over three decades in Olmsted County, 
Minnesota. Clin Gastroenterol Hepatol. 2009;7(10):105561. doi: 10.1016/j.cgh.2009.06.023.

9. Straumann A, Simon HU. Eosinophilic esophagitis: escalating epidemiology? J Allergy Clin Immunol. 2005;115(2):418-9. doi: 10.1016/j.jaci.2004.11.006.

10. Hruz P, Straumann A, Bussmann C, Heer P, Simon HU, Zwahlen $\mathrm{M}$, et al. Escalating incidence of eosinophilic esophagitis: a 20-year prospective, population-based study in Olten County, Switzerland. J Allergy Clin Immunol. 2011;128(6):1349-1350.e5. doi: 10.1016/j.jaci.2011.09.013.

11. Cherian S, Smith NM, Forbes DA. Rapidly increasing prevalence of eosinophilic oesophagitis in Western Australia. Arch Dis Child. 2006;91(12):1000-4. doi: 10.1136/ adc.2006.100974.

12. Whitney-Miller CL, Katzka D, Furth EE. Eosinophilic esophagitis: a retrospective review of esophageal biopsy specimens from 1992 to 2004 at an adult academic medical center. Am J Clin Pathol. 2009;131(6):788-92. doi: 10.1309/ AJCPOMPXJFP7EB4P.

13. Noel RJ, Putnam PE, Rothenberg ME. Eosinophilic esophagitis. N Engl J Med. 2004;351(9):940-1. doi: 10.1056/ NEJM200408263510924.

14. Dellon ES, Jensen ET, Martin CF, Shaheen NJ, Kappelman MD. Prevalence of eosinophilic esophagitis in the United States. Clin Gastroenterol Hepatol. 2014;12(4):589-96.e1. doi: 10.1016/j.cgh.2013.09.008.

15. Mansoor E, Cooper GS. The 2010-2015 Prevalence of Eosinophilic Esophagitis in the USA: A Population-Based Study. Dig Dis Sci. 2016;61(10):2928-2934. doi: 10.1007/ s10620-016-4204-4.

16. Furuta GT, Liacouras CA, Collins MH, Gupta SK, Justinich C, Putnam PE, et al. Eosinophilic esophagitis in children and adults: a systematic review and consensus recommendations for diagnosis and treatment. Gastroenterology. 2007;133(4):1342-63. doi: 10.1053/j.gastro.2007.08.017.

17. Liacouras CA, Spergel J, Gober LM. Eosinophilic esophagitis: clinical presentation in children. Gastroenterol Clin North Am. 2014;43(2):219-29. doi: 10.1016/j. gtc.2014.02.012.

18. Dellon ES, Gonsalves N, Hirano I, Furuta GT, Liacouras CA, Katzka DA, et al. ACG clinical guideline: Evidenced based approach to the diagnosis and management of esophageal eosinophilia and eosinophilic esophagitis (EoE). Am J Gastroenterol. 2013;108(5):679-92. doi: 10.1038/ ajg.2013.71.

19. Konikoff MR, Noel RJ, Blanchard C, Kirby C, Jameson SC, Buckmeier BK, et al. A randomized, double-blind, placebocontrolled trial of fluticasone propionate for pediatric eosinophilic esophagitis. Gastroenterology. 2006;131(5):138191. doi: 10.1053/j.gastro.2006.08.033.

20. Dohil R, Newbury R, Fox L, Bastian J, Aceves S. Oral viscous budesonide is effective in children with eosinophilic esophagitis in a randomized, placebo-controlled trial. Gastroenterology. 2010;139(2):418-29. doi: 10.1053/j. gastro.2010.05.001.
21. Straumann A, Conus S, Degen L, Felder S, Kummer $\mathrm{M}$, Engel $\mathrm{H}$, et al. Budesonide is effective in adolescent and adult patients with active eosinophilic esophagitis. Gastroenterology. 2010;139(5):1526-37, 1537.e1. doi: 10.1053/j.gastro.2010.07.048.

22. Straumann A, Spichtin HP, Grize L, Bucher KA, Beglinger C, Simon HU. Natural history of primary eosinophilic esophagitis: a follow-up of 30 adult patients for up to 11.5 years. Gastroenterology. 2003;125(6):1660-9.

23. Dellon ES. Epidemiology of eosinophilic esophagitis. Gastroenterol Clin North Am. 2014;43(2):201-18. doi: 10.1016/j.gtc.2014.02.002.

24. Spergel JM, Brown-Whitehorn TF, Beausoleil JL, Franciosi J, Shuker M, Verma R, et al. 14 years of eosinophilic esophagitis: clinical features and prognosis. J Pediatr Gastroenterol Nutr. 2009;48(1):30-6. doi: 10.1097/ MPG.0b013e3181788282.

25. DeBrosse CW, Franciosi JP, King EC, Butz BK, Greenberg $\mathrm{AB}$, Collins $\mathrm{MH}$, et al. Long-term outcomes in pediatriconset esophageal eosinophilia. J Allergy Clin Immunol. 2011;128(1):132-8. doi: 10.1016/j.jaci.2011.05.006.

26. Orenstein SR, Shalaby TM, Di Lorenzo C, Putnam PE, Sigurdsson L, Mousa H, et al. The spectrum of pediatric eosinophilic esophagitis beyond infancy: a clinical series of 30 children. Am J Gastroenterol. 2000;95(6):1422-30. doi: 10.1111/j.1572-0241.2000.02073.x.

27. Pierre R, Guisande A, Sifontes L, Sosa P, Ninomiya I, González L, et al. Diagnóstico y tratamiento de la esofagitis eosinofílica en niños. Revisión de la literatura y recomendaciones basadas en la evidencia. Acta Gastroenterol Latinoam. 2015;45:263-71.

28. Straumann A, Spichtin HP, Bucher KA, Heer P, Simon HU. Eosinophilic esophagitis: red on microscopy, white on endoscopy. Digestion. 2004;70(2):109-16. doi: 10.1159/000080934.

29. Hurrell JM, Genta RM, Melton SD. Histopathologic diagnosis of eosinophilic conditions in the gastrointestinal tract. Adv Anat Pathol. 2011;18(5):335-48. doi: 10.1097/ PAP.0b013e318229bfe2.

30. Grin A, Streutker CJ. Esophagitis: old histologic concepts and new thoughts. Arch Pathol Lab Med. 2015;139(6):7239. doi: 10.5858/arpa.2014-0132-RA.

31. Kapel RC, Miller JK, Torres C, Aksoy S, Lash R, Katzka DA. Eosinophilic esophagitis: a prevalent disease in the United States that affects all age groups. Gastroenterology. 2008; 134(5):1316-21. doi: 10.1053/j.gastro.2008.02.016.

32. Franciosi JP, Tam V, Liacouras CA, Spergel JM. A casecontrol study of sociodemographic and geographic characteristics of 335 children with eosinophilic esophagitis. Clin Gastroenterol Hepatol. 2009;7(4):415-9. doi: 10.1016/j. cgh.2008.10.006.

33. Pierre R, Vieira M, Vázquez-Frías R, Nimoniya I, Messere G, Daza W, et al. Estudio multicéntrico sobre la epidemiologia de la esofagitis eosinofílica pediátrica en América Latina. Rev Gen. 2016;70:125-30. 
34. DeBrosse CW, Case JW, Putnam PE, Collins $\mathrm{MH}$, Rothenberg ME. Quantity and distribution of eosinophils in the gastrointestinal tract of children. Pediatr Dev Pathol. 2006;9(3):210-8. doi: 10.2350/11-05-0130.1.

35. Collins $\mathrm{MH}$. Histopathologic features of eosinophilic esophagitis and eosinophilic gastrointestinal diseases. Gastroenterol Clin North Am. 2014;43(2):257-68. doi: 10.1016/j.gtc.2014.02.007.

36. Aceves SS, Newbury RO, Dohil R, Bastian JF, Broide DH. Esophageal remodeling in pediatric eosinophilic esophagitis. J Allergy Clin Immunol. 2007;119(1):206-12. doi: 10.1016/j.jaci.2006.10.016.

37. Blaser MJ. Helicobacter pylori and esophageal disease: wake-up call? Gastroenterology. 2010;139(6):1819-22. doi: 10.1053/j.gastro.2010.10.037.
38. Dellon ES, Peery AF, Shaheen NJ, Morgan DR, Hurrell JM, Lash RH, et al. Inverse association of esophageal eosinophilia with Helicobacter pylori based on analysis of a US pathology database. Gastroenterology. 2011;141(5):1586-92. doi: 10.1053/j.gastro.2011.06.081.

39. Furuta K, Adachi K, Aimi M, Ishimura N, Sato S, Ishihara $\mathrm{S}$, et al. Case-control study of association of eosinophilic gastrointestinal disorders with Helicobacter pylori infection in Japan. J Clin Biochem Nutr. 2013;53(1):60-2. doi: 10.3164/jcbn.13-15.

40. Chen Y, Blaser MJ. Inverse associations of Helicobacter pylori with asthma and allergy. Arch Intern Med. 2007;167(8):821-7. doi: 10.1001/archinte.167.8.821. 